\title{
Axial Non-linear Dynamic Soil-Pile Interaction - Keynote
}

\author{
A. Holeyman ${ }^{1}$ and V. Whenham ${ }^{2}$ \\ ${ }^{1}$ Université catholique de Louvain, iMMC, GeoMEM, 1348 Louvain-la-Neuve, Belgium \\ ${ }^{2}$ Fugro Geoconsulting Belgium, 1150 Brussels, Belgium
}

\begin{abstract}
This keynote lecture describes recent analytical and numerical advances in the modeling of the axial nonlinear dynamic interaction between a single pile and its embedding soil. On one hand, analytical solutions are developed for assessing the nonlinear axial dynamic response of the shaft of a pile subjected to dynamic loads, and in particular to vibratory loads. Radial inhomogeneity arising from shear modulus degradation is evaluated over a range of parameters and compared with those obtained by other authors and by a numerical radial discrete model simulating the pile and soil movements from integration of the laws of motion. New approximate non linear solutions for axial pile shaft behaviour developed from general elastodynamic equations are presented and compared to existing linear solutions. The soil non linear behaviour and its ability to dissipate mechanical energy upon cyclic loading are shown to have a significant influence on the mechanical impedance provided by the surrounding soil against pile shaft movement. The limitations of over-simplified modelling of pile response are highlighted.
\end{abstract}

\section{Introduction}

\subsection{Typical situations}

Piles are used to support civil engineering structures whenever loads are concentrated relative to the soil bearing capacity and thus strength. They consist of elongated structural elements that are embedded in the soil to a depth that will allow bearing layers to develop a safe resistance. Typical situations where piles are used are illustrated on figure 1 . In the most common case referred to as "active" pile, the pile head receives the load from the superstructure and transmits it to the resisting soil.

Although all 6 load components (forces components and moments about 3 orthogonal axes) need to be considered, the pile axial component generally governs the design of the pile and in particular its embedment depth . In addition to static loads resulting from gravity, operational transient or periodic loads may warrant special design requirements. In response to the quickly expanding market of offshore renewable energy [1], piles have been recently used in several configurations to support wind turbines, as illustrated in figure 1 .

Extreme loading cases have also to be considered due to the fact that piles have to be installed at depth: such cases involve pile impact driving as well as vibratory driving.

Due to space and time constraints, this lecture will only focus on the axial response of a pile subjected to a
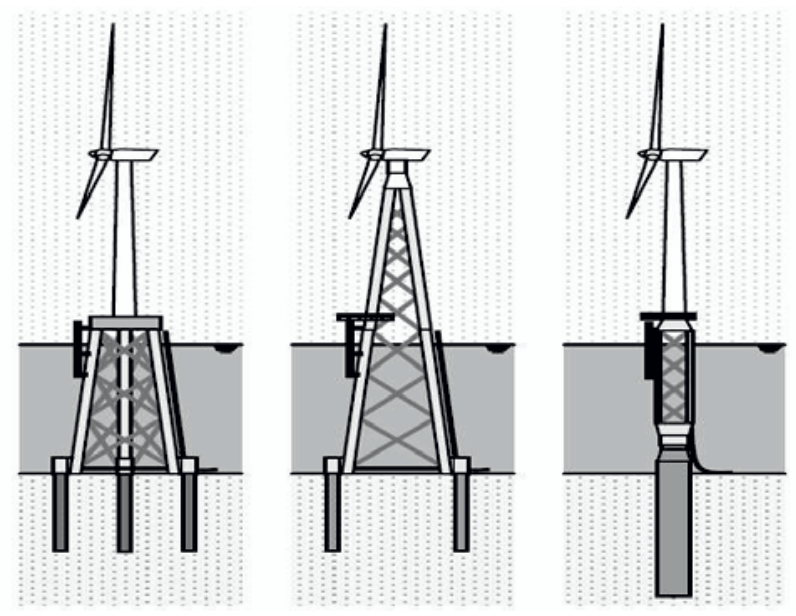

Fig.1. Typical use of piles to support wind turbines: Tripod, Jacket, and Monopile [1]

harmonic axial load. Similar contributions can be developed on the pile lateral response, torsional response and would justify a discussion on the coupling. Finally, most of the discussion will revolve about the friction component of the pile resistance, leaving out the singularity brought about by the pile bottom end.

Whatever the mode considered, the engineering picture needs to involve three main ingredients: the complete definition of all actions on the pile, the structural characterization of the pile, and the characterization of the embedding medium, i. e. the soil. 


\subsection{Pile characterization}

Piles used for large projects are commonly made of concrete or steel. Generally concrete is used for onshore applications while steel is used for offshore applications. In the later case, steel pipes are used to construct "pipe piles". The advantage of that geometry is that the volume of soil to be displaced to accommodate pile insertion into the soil is a very small fraction of the gross pile volume that will govern the geotechnical capacity of the pile. For large diameter piles, the inertia of the soil volume inside the pipe will prevent the soil core from moving down in unison with the pile during driving, leading to what is termed a "coring" mode of driving.

Most tubular piles are driven by impact using special pieces of equipment (hydraulic pile hammers) set on top of the pile during installation. More rarely, piles can be vibrated into the ground using vibratory hammers. Once installed to an appropriate depth, a pile will develop its bearing capacity over time, moving from its end-ofinstallation capacity towards its long-term capacity. The pile geotechnical capacity will come from contact stresses generated by the soil along two interfaces: a shear stress along the pile lateral surface (called shaft) and a normal stress against the pile end bearing area (called toe).

The axial bearing performance of a pile can be characterized by its response under axial static loading. As illustrated in figure 2a, the pile load-settlement curve provides the overall relationship between the applied load $F$ and the pile settlement $s$. One can notice that under limited loads, the pile responds linearly, but endures non recoverable displacements under larger loads, with the ultimate limit state being defined by unlimited displacements under an asymptotic load. This reflects the non linear behaviour of the soil while the pile material remains well within its elastic realm. This forces us to address an essential feature of the system, namely the soil behaviour that will be characterized in Section 2.

\subsection{Simplified Soil-Pile interaction}

Engineering methods that are commonly applied to assess pile response under a static load applied at the head of the pile treat the pile as a column collecting reactions along its shaft and at the pile toe. Assuming that the embedding medium is elastic, it would appear possible to model each local reaction as proportional to the local displacement. Further assuming that each equivalent spring is independent from its neighbours allows one to treat each soil layer with its own properties.

This simplification known as a "Winkler" model has been extended to cope with non linear behaviour, leading to what is known in the geotechnical jargon as "t-z" and "Q$\mathrm{z}$ " curves. A " $\mathrm{t}-\mathrm{z}$ " curve models the non linear development of the local shear stress along the pile shaft versus local vertical displacement while a "Q-z" curve models the non linear development of the pile toe resistance versus the pile base displacement. figure $2 b$ schematizes such simplified modelling of the soil-pile interaction.

By extending Winkler approach initially developed in the static domain, the dynamic response of pile shafts embedded in an elastic medium can be studied by replacing the soil surrounding the pile with a series of independent springs and dashpots. Notably, Smith [2] used that approach to model soil resistance to pile driving, leading to the emergence of soil-pile dynamic interaction parameters known as "quake" and "damping", as illustrated as Q and J on figure 3.
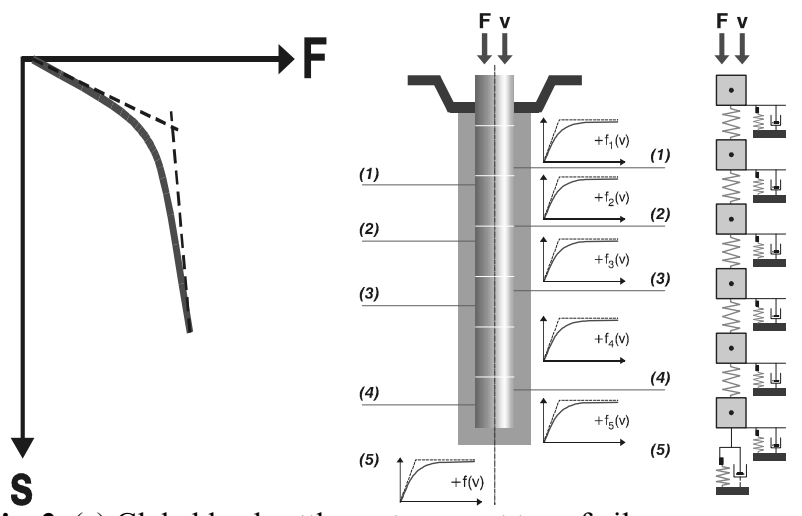

Fig. 2. (a) Global load-settlement curve at top of pile (b) Embedded pile as continuously supported column

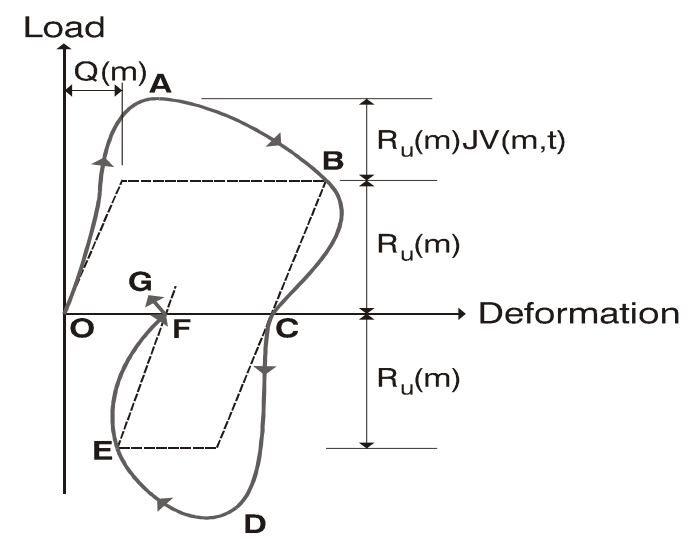

Fig. 3. Smith visco-elasto-plastic load-deformation curve for local slice of pile shaft (adapted from [2])

For a pile undergoing harmonic axial motion, coefficients of the Winkler springs and dashpots depend on the frequency. Such coefficients can be obtained by considering the elastodynamic problem of an infinitely long pile subjected to harmonic vertical displacements.

An alternative approach consists in modelling fully coupled 3D pile-soil interactions, for example by means of the finite element method. However because of the complexity of the problem, especially when the pile is subjected to high strain loading conditions, this approach makes it difficult to properly simulate the essential phenomena at play and is faced with the challenge to harness adequate model parameters. Practical use of the full 3D finite element approach is further hampered by its high demand for computer resources.

While structural engineers are quite fond of simplifications of the pile behaviour boiling it down to a 
single spring and sometimes a dashpot, they tend to overlook that a pile is a complex infrastructural system that interacts with the soil surrounding and with the superstructure. Furthermore soil is a medium that is far from behaving linearly, as can be summarized below.

\section{Soil characterization}

Soil is a multiphase medium made of solid particles whose composite behaviour depends on many factors: attributes of particles, fluid filling the voids left between the solids, stress history, just to name a few. While the particles can be characterized by their nature, size, and shape, their overall behaviour with respect to the water content can be characterized by their plasticity index (PI). The PI of sand is zero while clay minerals can exhibit values in excess of 50 , with silts having intermediate values.

Volume variations and distortion of soil depend solely on the soil "effective" stress, i.e. the stress between particles, the pore pressure having no intrinsic role other than taking a part of the total stress. One rather unique feature of soil behaviour is its tendency to change volume when sheared. Loosely packed soils tend to contract while densely packed soils tend to dilate. Soil shear strength essentially comes from friction, which is controlled by effective stress while the effective internal friction angle generally assumes a value close to $30^{\circ}$.

When saturated with water, a contractant assemblage of particles can only modify its volume inasmuch water has the time to drain away from it. This means that low permeability soils or soils undergoing fast loading have to deform without changing their volume, which implies a substantial change in their effective stress. Such a volumetric constraint explains why the strength of a soil depends so drastically upon its loading rate. At one extreme, loading is so slow that volume changes can be accommodated without any interference from the pore fluid, and the soil will behave as "drained". At the other extreme, loading is so fast that volume remains constant and the material will behave as undrained.

Whether drained or undrained, the latter case being more common under dynamic loading, soil beahvior exhibits several features that are characterized in the following section, namely, stiffness, strain hardening and yield criteria, implying material damping upon cyclic loading.

\subsection{Key attributes of soil behavior}

\subsubsection{Small strain stiffness}

An initial (or maximum) shear modulus can be calculated for rounded grained sands using the relation of Hardin and Black [3]:

$G_{\max }=6908 \frac{(2.17-e)^{2}}{1+e} \sigma^{0.5}[\mathrm{kPa}]$ where $e$ is the soil void ratio and $\sigma^{\prime}$ is the effective confining mean stress usually calculated as:

$\sigma^{\prime}=\frac{\sigma_{v}^{\prime}+2 \sigma_{r, 0}^{\prime}}{3}$

Where $\sigma_{v}^{\prime}$ is the easily calculated effective initial vertical stress, $\sigma_{r, 0}^{\prime}=k \sigma_{v}^{\prime}$ is the effective horizontal stress, with $k$ being the coefficient of horizontal stress in the soil. For a "wished-in-place" pile assumption, an at rest coefficient is estimated herein using Jacky's formula $k=1-\sin \varphi^{\prime}$ wherein $\varphi^{\prime}$ is the soil internal friction angle.

\subsubsection{Strain hardening}

Thanks to numerous forms of soil testing, the relationship between shear stress and shear strain has been shown to deviate from the initial tangent value $G_{\max }$ defined above as shear strain increases, as shown in figure 4 . This can be viewed as "strain hardening" since the shear stress increases beyond an "elastic" limit to be identified. In soil mechanics, this feature is preferably described in terms of shear modulus degradation [4] inasmuch the secant modulus $G_{s}$ degrades with strain. Two examples of models commonly accepted to characterize the shear modulus degradation are discussed in Sections 2.2 and 2.3. Densely packed soils, such as stiff clays can also exhibit some form of strain softening, which will not be covered in this lecture.

At large strains, the soil reaches a "critical state" shear strength characterized by a constant volume and mean stress. That ultimate limit state will be characterized by the value of $\tau_{\max }$ shown on figure 4 .

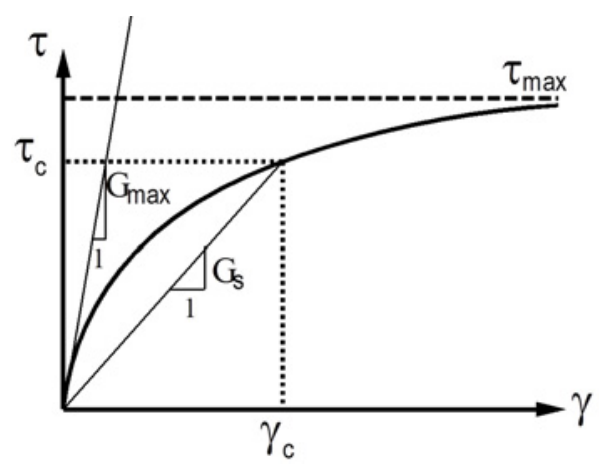

Fig. 4. Hyperbolic shear stress - shear strain model for soils [4]

\subsubsection{Material damping and viscous equivalent}

According to Masing rules [5] which will be deemed applicable, if the loading curve is characterized by the relationship $f(F, \delta)=0$ with $F$ the load and $\delta$ the displacement, then for a cycle between points $\left(\delta_{c}, F_{c}\right)$ and $\left(-\delta_{c},-F_{c}\right)$, the loading-unloading curves are: 
$f\left(\frac{\delta-\delta_{c}^{*}}{2}, \frac{F-F_{c}^{*}}{2}\right)=0$

where $\left(\delta_{c}^{*}, F_{c}^{*}\right)$ is the point of loading inversion. figure 5 provides an illustration of Masing's rules, showing how the backbone loading curve can be expanded and rotated to generate the unloading and reloading curves.

The loop developed within this stress-strain plane highlights the dissipation of mechanical energy during a complete loading cycle. Such a material dissipation is characterized by the soil "damping coefficient", a relative measure of the dissipated energy $\Delta W$ within one cycle to the maximum accumulated elastic energy. If $\tau_{c}$ is the amplitude of the shear stress and $\gamma_{c}$ the amplitude of the shear strain, the soil damping coefficient is defined as $\xi=\Delta W /(4 \pi W)$, with $W=\gamma_{c} \cdot \tau_{c} / 2$.

It should be noted that for a given $\tau_{\text {max }}$ the material damping depends on $\gamma_{c}$ but not on the frequency of a potentially considered harmonic movement. Harmonic displacements prescribed by the pile generate cyclic deformations and stresses within the influenced soil zone that can be conveniently expressed by:

$\tau=\tau_{c} \cdot e^{i \omega t}=G \cdot(1+2 i \xi) \cdot \gamma_{c} \cdot e^{i \omega t}=G^{*} \cdot \gamma_{c} \cdot e^{i \omega t}$

wherein $G^{*}$ is the complex shear modulus.

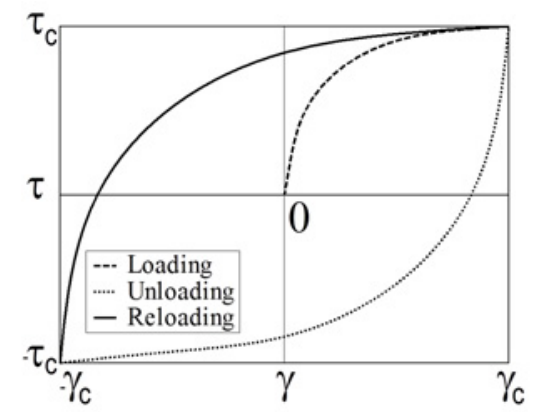

Fig. 5. Masing's rules [5] applied to stress-strain curves

Such an expression postulates that energy losses can be attributed to an out of phase term treating the soil as visco-elastic. In that case, a so called "linear equivalent" soil model is invoked, although strictly speaking, it is linear only under static conditions.

Assuming hysteretic energy losses can be handled at a given frequency by an equivalent viscous-elastic shear modulus $G^{*}=G .(1+2 i \xi)$, the equivalent soil viscosity can be expressed under harmonic conditions at frequency $\omega$ as $\eta=\partial \tau / \partial \dot{\gamma}=2 G \xi / \omega$, with $\dot{\gamma}$ being the shear strain rate. Although it does not respect the shape of the stress-strain loop, the assumption of the "linear equivalent" medium is generally accepted because of its mathematical convenience, as will be shown in Section 3.3 .

\subsubsection{Cyclic degradation and liquefaction}

Beyond strain related shear modulus "degradation" discussed above, soil is subject to fatigue whenever strain cycles of sufficient amplitude accumulate. In loose granular soils that are saturated, the cyclic degradation can be compounded by the onset of "liquefaction". This phenomenon occurring in contractive materials involves the increase of the pore pressure to the point that effective stresses vanish. Since soil strength is intrinsically related to friction, the removal of any effective stress actually transforms the soil into a medium unable to resist to shear stress, i.e. a fluid.

\subsection{Hardin and Drnevich [4] model}

The relationship between shear stress and shear strain under undrained conditions can be assumed to follow the soil model suggested by Hardin \& Drnevich [4] and based on Kondner [6] formulation.

$$
\tau=\frac{\gamma}{1 / G_{\max }+\gamma / \tau_{\max }}=\tau_{\max } \cdot \frac{\gamma}{\gamma+\gamma_{r}}
$$

where $\tau$ is the shear stress, $\gamma$ is the shear strain, $\tau_{\max }$ is the maximal shear stress (shear strength), $G_{\max }$ is the initial shear modulus and $\gamma_{r}=\tau_{\max } / G_{\max }$ is the reference strain. Degradation of the secant shear modulus with the shear strain can therefore be defined by:

$$
G=\frac{\tau}{\gamma}=\frac{\tau_{\max }}{\gamma+\gamma_{r}}=G_{\max } \cdot \frac{\gamma_{r}}{\gamma+\gamma_{r}}=G_{\max }\left(1-\frac{\tau}{\tau_{\max }}\right)
$$

Applying Masing's rules to the Hardin \& Drnevich [4] loading curve leads to the following expression of the damping coefficient as a function of $\gamma_{c}$ :

$$
\xi=\frac{2}{\pi} \cdot\left(2 \frac{\gamma_{r}}{\gamma_{c}^{2}} \cdot\left(\gamma_{r}+\gamma_{c}\right) \cdot \ln \left(\frac{\gamma_{r}}{\gamma_{r}+\gamma_{c}}\right)+2 \frac{\gamma_{r}}{\gamma_{c}}+1\right)
$$

Illustrations of the Hardin \& Drnevich [4] formulation and Masing's rules [5] are provided in figures 4 to 6 .

\subsection{Ishibashi and Zhang [7] model}

Alternatively, the stress-strain relation suggested by Ishibashi and Zhang [7] can be considered to characterize soil nonlinearity, especially in the weakened zone close to the pile shaft. The degradation of the secant shear modulus is expressed as a function of the shear strain $\gamma$, of the effective confining mean stress $\sigma^{\prime}$, and of the plasticity index IP: 
$\frac{G}{G_{\max }}=0.5\left\{1+\tanh \left(0.492 \ln \frac{0.000102+n}{\gamma}\right)\right\} \sigma^{\prime m}$

where

$m=0.272\left\{1-\tanh \left(0.4 \ln \frac{0.000556}{\gamma}\right)\right\} e^{-0.0145 I P^{1.3}}$

$n= \begin{cases}0.0 & \text { for } \quad \text { IP }=0 \\ 3.37 \times 10^{-6} I P^{1.404} & \text { for } \quad 0<\mathrm{IP} \leq 15 \\ 7 \times 10^{-7} I P^{1.976} & \text { for } \quad 15<\mathrm{IP} \leq 70 \\ 2.7 \times 10^{-5} I P^{1.115} & \text { for } \quad \mathrm{IP}>70\end{cases}$

The hysteretic damping coefficient resulting from equations 7a, b, c can be expressed as [7]:

$\xi=\frac{1+e^{-0.0145 I P^{1.3}}}{6}\left[0.586\left(\frac{G}{G_{\max }}\right)^{2}-1.547 \frac{G}{G_{\max }}+1\right]$

The asymptotic value of $\xi$ depends on the plasticity index $\left(\xi_{I P}^{\max }=\frac{1+e^{-0.0145 I P^{1.3}}}{6}\right)$, reaching a maximum of $33 \%$ for $\mathrm{IP}=0$ and $18.3 \%$ for $\mathrm{IP}=50$. This feature makes the Ishibashi and Zhang's model more flexible than the basic hyperbolic model [4] and applicable to several types of soils according to their plasticity attributes.

\subsection{Experimental evidence and complicating factors}

Figure 6 presents a comparison between experimental curves established by Vucetic and Dobry [8] for soils of varying PI and the above theoretical formulations of the damping coefficient (6 and 8), for various reference strains. It can be noted that experimental values of $\xi$ can typically range between 0 and 0.4 .

In soils that are subject to cyclic degradation, such as loose sands or sensitive clays, the maximum shear stress (shear strength) $\tau_{\max }$ evolves as cycles accumulate. Such an evolution is not explicitly accounted for in the analytical models presented hereafter; rather it is accounted in the choice of an equivalent $\tau_{\max }$ that takes

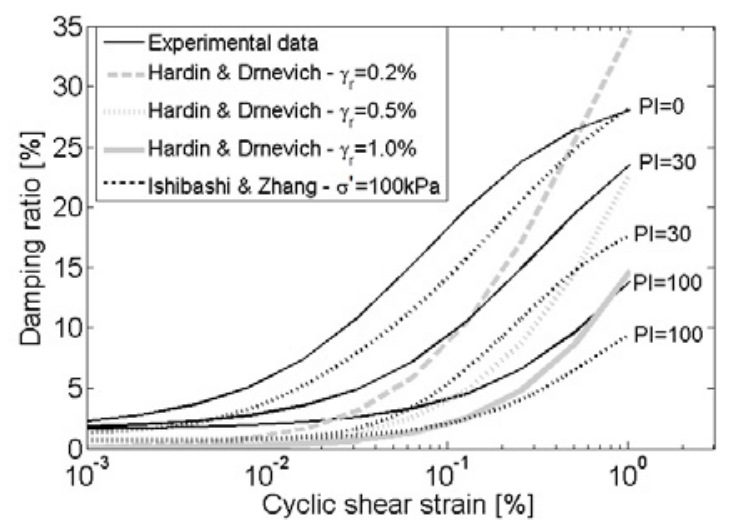

Fig. 6. Soil damping coefficient $\xi$ as a function of cyclic shear strain $\gamma_{c}$ : experimental curves vs. models [4] and [7] a representative number of cycles and degradability into account. That refinement can however be explicitly accounted for in the numerical models developed by Holeyman [9], where several degradation laws of $\tau_{\max }$ are implemented according to the local shear strain history.

\subsection{More advanced models}

Many more models have been developed by researchers attempting to capture various features of the complex soil behaviour. These can be approached by separating recoverable and non recoverable deformations, the latter being handled through plastic theory. Three-dimensional representations of the yield function in the stress space and the choice of flow rules in the strain space are then necessary, requiring the knowledge of up to tens of parameters that are difficult to determine experimentally for many engineering projects.

The separation between elastic and plastic domains can be circumvented by the use of so-called "Hypoplasticity", which appears to gain popularity thanks to a more reasonable number of parameters. While the initial hypoplastic model [10] required 8 parameters, those incorporating the intergranular concept and cyclic features [11] can go up to 13 . That number contrasts with the more manageable 2 or 3 parameters necessary to understand what are believed to be the essentials of pile response used in the remainder of this invited lecture. Moreover the basic parameters used ( $e$ and $P I$ or $G_{\max }$ and $\tau_{\max }$ ) are standard geotechnical parameters widely available from customary site characterization.

\section{Problem statement and linear solution}

\subsection{Idealized conditions}

The problem considered in this keynote lecture involves a vertical cylindrical floating pile shaft of infinite length and rigidity, embedded within an infinite homogeneous soil medium. The pile shaft is subjected to a purely harmonic axial displacement prescribed by $w_{0}=w_{0 c} \cdot \cos \omega t$ where $w_{0 c}$ is the amplitude of displacement of the pile shaft, and $\omega$ is the circular frequency, and $t$ the time. The examined system is a unit slice of the problem as shown on figure 7, isolating a single pile shaft segment and associated unit thickness soil layer of infinite radial extent. Plane strain conditions prevail across any horizontal slice because of the infinite extent of the considered problem in the axial direction and uniformity of the prescribed movement along the vertical direction. The layer outside the pile can be viewed as an infinite shear plate with a circular hole about which the harmonic vertical motion is prescribed.

The prescribed dynamic displacement generates cyclic deformations and stresses within the analyzed soil 


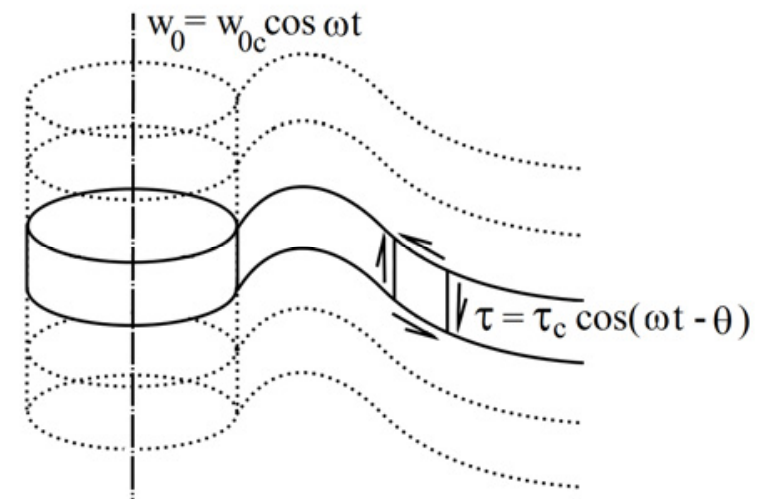

Fig. 7. Unit layer considered within infinite pile shaft and embedding soil

layer shown on figure 7 that can be represented by $\tau_{c} \cdot \cos (\omega t-\theta)$ where $\tau_{c}$ is the stress at radial distance $\mathrm{r}$ and $\theta$ is the phase difference with respect to the displacement applied at the soil-shaft interface.

In practice, stress anisotropy induced due to the weight of the soil will result in a specific distribution of the shear modulus with depth. Furthermore, soil layering is not homogeneous as the pile can endure axial compression, making the infinite extent of the pile and surrounding soil assumption less legitimate. Averaging of soil properties along the depth of the pile shaft should be considered prior to using a single layer model.

Relationship between shear stress and shear strain under undrained conditions will be assumed to follow the soil model suggested by Hardin \& Drnevich [4] and based on Kondner [6] formulation.

\subsection{Soil impedance to pile shaft movement}

Under the assumption of small deformations and absence of slippage at the pile shaft-soil interface, and provided radial deformations as well as the pile mass effect can be neglected in the analysis, the differential equation describing the vertical motion $w(r, t)$ of a floating rigid pile shaft embedded in a homogeneous isotropic elastic soil medium of shear modulus $G$ and volumetric mass $\rho$ is given by:

$G \frac{\partial^{2} w}{\partial r^{2}}+\left(\frac{G}{r}+\frac{\partial G}{\partial r}\right) \cdot \frac{\partial w}{\partial r}=\rho \cdot \frac{\partial^{2} w}{\partial t^{2}}$

Let us consider further that the vertical movement is harmonic and stationary; it can be characterized by the following relationship: $w(r, t)=w_{c} \cdot e^{i \omega t}$, where $w_{c}$ is the amplitude of the soil displacement that solely depends on the radial distance r. Assuming hysteretic energy losses can be handled at a given frequency by an equivalent viscous-elastic shear modulus $G^{*}=G .(1+2 i \xi)$, the equation of movement becomes:

$$
G^{*} \frac{\partial^{2} w}{\partial r^{2}}+\left(\frac{G^{*}}{r}+\frac{\partial G^{*}}{\partial r}\right) \cdot \frac{\partial w}{\partial r}=\rho \cdot \frac{\partial^{2} w}{\partial t^{2}}
$$

Extending the Winkler concept introduced in Section 1.3, the dynamic reaction of the soil surrounding the pile shaft may be expressed with reference to an equivalent springdashpot system anchored to a stationary point, as illustrated by figure 8 . The dynamic soil reaction opposing the prescribed pile shaft movement can then be expressed as

$$
P_{z}(r, t)=C_{z} \cdot \dot{w}(r, t)+K_{z} \cdot w(r, t)
$$

with $P_{z}$ the soil reaction per unit length of shaft, $C_{z}$ the damping coefficient and $K_{z}$ the stiffness coefficient. Since the problem has been stated within a unit thickness soil layer, it should be noted that $P_{z}, C_{z}$, and $K_{z}$ are expressed per unit length of pile shaft, and thus typically in the following respective units $[k N / m],[k P a / s]$, and $[k P a]$.

Assuming $P_{z}(r, t)$ is harmonic, we can define the unit (lineal) shear impedance of the soil against the pile shaft movement in the $\mathrm{z}$ direction as:

$$
\begin{aligned}
& I_{z}=\frac{P_{z c}}{w_{c}}=\left(C_{z} \cdot i \cdot \omega+K_{z}\right) \\
& =G_{s 0}\left(C_{z a} \cdot i+K_{z a}\right)=\frac{2 \pi \cdot r_{0}}{w_{c}\left(r_{0}\right)} \cdot \tau_{c}\left(r_{0}\right)
\end{aligned}
$$

with $G_{s 0}$ the shear modulus at the pile shaft-soil interface $\left(r=r_{0}\right)$, and

$$
\begin{aligned}
& K_{z a}=\mathfrak{R}\left\{I_{z}\right\} / G_{s 0} \\
& C_{z a}=\mathfrak{I}\left\{I_{z}\right\} /\left(\omega \cdot G_{s 0}\right)
\end{aligned}
$$

the dimensionless stiffness and damping parameters.

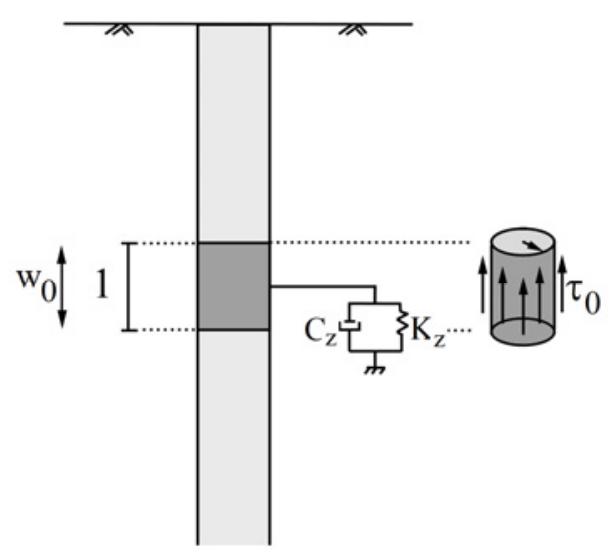

Fig. 8. Equivalent Winkler spring-dashpot soil model supporting the pile shaft 


\subsection{Analytical solution for equivalent medium}

Assuming that the shear modulus is independent of the radial distance $r$ to the pile shaft, a unique shear wave velocity can be defined as: $V_{s}=\sqrt{G_{s} / \rho}$ for a purely elastic medium (or $V_{s}^{*}=\sqrt{G_{s}^{*} / \rho}$ for the equivalent visco-elastic medium) (10) can be thus expressed as:

$\frac{\partial^{2} w_{c}}{\partial r^{2}}+\frac{1}{r} \cdot \frac{\partial w_{c}}{\partial r}+\left(k^{*}\right)^{2} \cdot w_{c}=0$

where $k=\omega / V_{s}$ is the shear wave number, the asterisk indicating that the viscous soil behavior (characterized by the Kelvin-Voigt formulation) is taken into consideration, i.e. $k^{*}=\omega / V_{s}^{*}$

Defining the dimensionless frequency for the pile shaft-soil interface as $a=\omega \cdot r_{0} / V_{s}$ or $a^{*}=\omega \cdot r_{0} / V_{s}^{*}$, the general solution of (14) is given by

$w_{c}=w_{0 c} \cdot H_{0}^{(2)}\left(a^{*} \cdot \frac{r}{r_{0}}\right) / H_{0}^{(2)}\left(a^{*}\right)$

with $H_{v}^{(2)}=J_{v}-i . Y_{v}$ the Hankel function and $J_{v}$, $Y_{v}$, Bessel functions of order $v$ of the first and second type, respectively. Based on the $\tau(r, t)=G^{*} . \partial w(r, t) / \partial r$ relationship, the solution can also be expressed in terms of stress amplitudes:

$\tau_{c}=G_{s} \cdot(1+2 i \xi) \cdot \frac{a^{*} \cdot w_{0 c}}{r_{0}} \cdot H_{1}^{(2)}\left(a^{*} \cdot \frac{r}{r_{0}}\right) / H_{0}^{(2)}\left(a^{*}\right)$

Particular solutions can be obtained by applying adequate boundary conditions. A first boundary condition corresponds to the imposed displacement at the pile shaftsoil interface, i.e. $w_{c}=w_{0 c}$ for $r=r_{0}$. The second boundary condition is a radiation condition imposing that the wave should only propagate away from the vibration source at the outer boundary $(w(r \rightarrow \infty, t)$, also known as "Sommerfeld" condition [12]).

$$
\begin{aligned}
& \rightarrow \bar{w}_{c}(r)=\sqrt{\mathfrak{R}^{2}\left\{w_{c}(r)\right\}+\mathfrak{J}^{2}\left\{w_{c}(r)\right\}} \\
& =w_{0 c} \sqrt{\frac{J_{0}^{2} \cdot\left(a \cdot \frac{r}{r_{0}}\right)+Y_{0}^{2} \cdot\left(a \cdot \frac{r}{r_{0}}\right)}{J_{0}^{2} \cdot(a)+Y_{0}^{2} \cdot(a)}} \\
& \rightarrow \bar{\tau}_{c}(r)=\sqrt{\mathfrak{R}^{2}\left\{\tau_{c}(r)\right\}+\mathfrak{I}^{2}\left\{\tau_{c}(r)\right\}} \\
& =G_{s} \cdot \frac{a \cdot w_{0 c}}{r_{0}} \cdot \sqrt{\frac{J_{1}^{2} \cdot\left(a \cdot \frac{r}{r_{0}}\right)+Y_{1}^{2} \cdot\left(a \cdot \frac{r}{r_{0}}\right)}{J_{0}^{2} \cdot(a)+Y_{0}^{2} \cdot(a)}}
\end{aligned}
$$

Using [Equ.8] we deduce the equivalent impedance:

$$
I_{z}=-2 \pi \cdot a^{*} \cdot G_{s}(1+2 i \xi) \cdot \frac{H_{1}^{(2)}\left(a^{*}\right)}{H_{0}^{(2)}\left(a^{*}\right)}
$$

Dimensionless impedance parameters defined by (12) and (13) are depicted in figure 9, emphasizing the influence of the hysteretic damping coefficient. In the absence of viscous damping $(\xi=0)$ only radiation or geometrical damping prevails. In that case, the stiffness parameter (real part $K_{z, a}$ of impedance) tends toward $\pi$ for increasing frequencies $(a \rightarrow \infty)$, per (9). Except for low $a$ values, the dimensionless damping term $C_{z, a}$ linearly increases with frequencies at a rate of $\Delta C_{z, a}=2 \pi \Delta a$. An increased hysteretic damping coefficient enhances the quasi-linear increase of the total (hysteretic and radiation) damping term, but decreases the in-phase stiffness component. Figure 9 also shows that $K_{z, a}$ can become equal to 0 at particular values of $a$ $\left(a_{\left(K_{z, a}=0\right)}\right)$, implying a potential for some form of 'resonance' effect. Influence of the hysteretic damping on the infinite annular shear plate apparent 'resonant' frequency can be appreciated in figure 10. The physical reason for that effect remains unclear, despite the fact that similar phenomena have been experimentally observed on model tests [13].
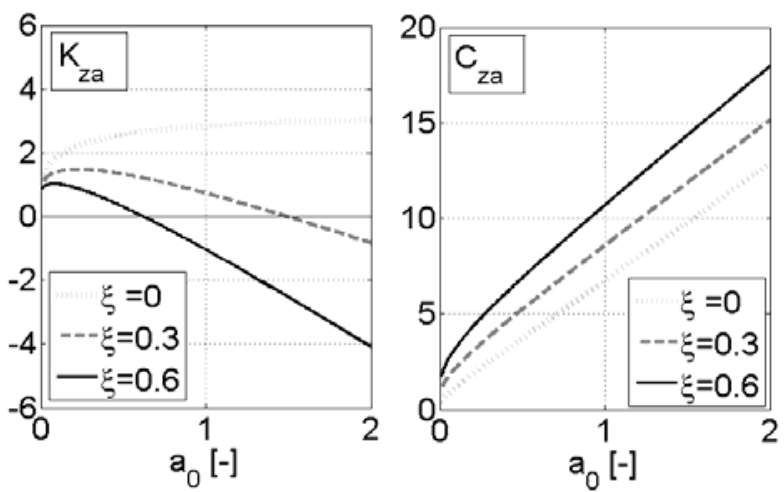

Fig. 9. Impedance parameters for homogeneous shear modulus

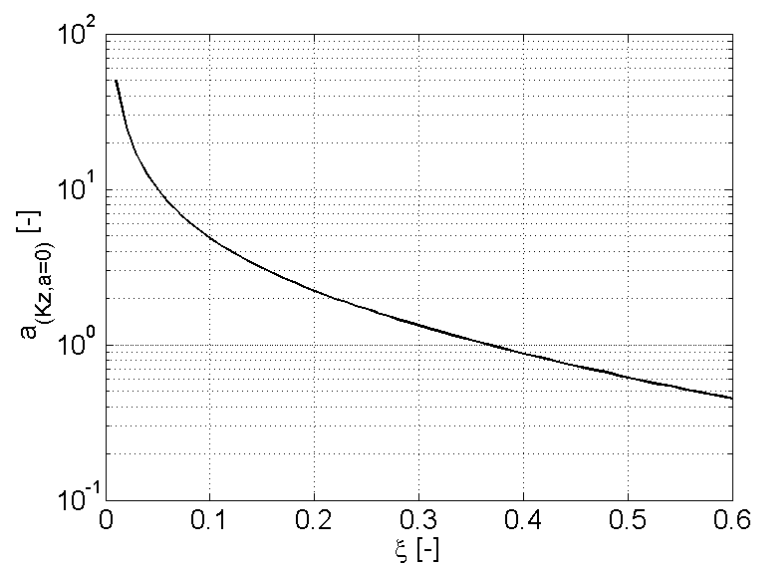

Fig. 10. Dimensionless "resonant" frequency of the soil annular shear plate vs. hysteretic damping coefficient 
Typical results expressed in terms of dimensionless displacement and stress amplitudes profiles are presented in figure 11 for two dimensionless frequency values and two damping coefficients. The dimensionless displacement (stress) amplitude is the ratio between the displacement (stress) amplitude $w_{c}\left(\tau_{c}\right)$ at a given radial distance to that $w_{0 c}\left(\tau_{0 c}\right)$ at the pile shaft-soil interface.

For $\xi=0$ and high $a$ values, displacement amplitudes attenuate according to the inverse of the square root of the radial distance, while for $\xi=0$ and low $a$ values, shear stress amplitudes attenuate according to the inverse of the radial distance. These results can be demonstrated by considering the asymptotic behavior of the Bessel functions for $a \rightarrow \infty$ (17) and (18). It can also be noted that material damping enhances the radial attenuation of both displacement and stress amplitudes.
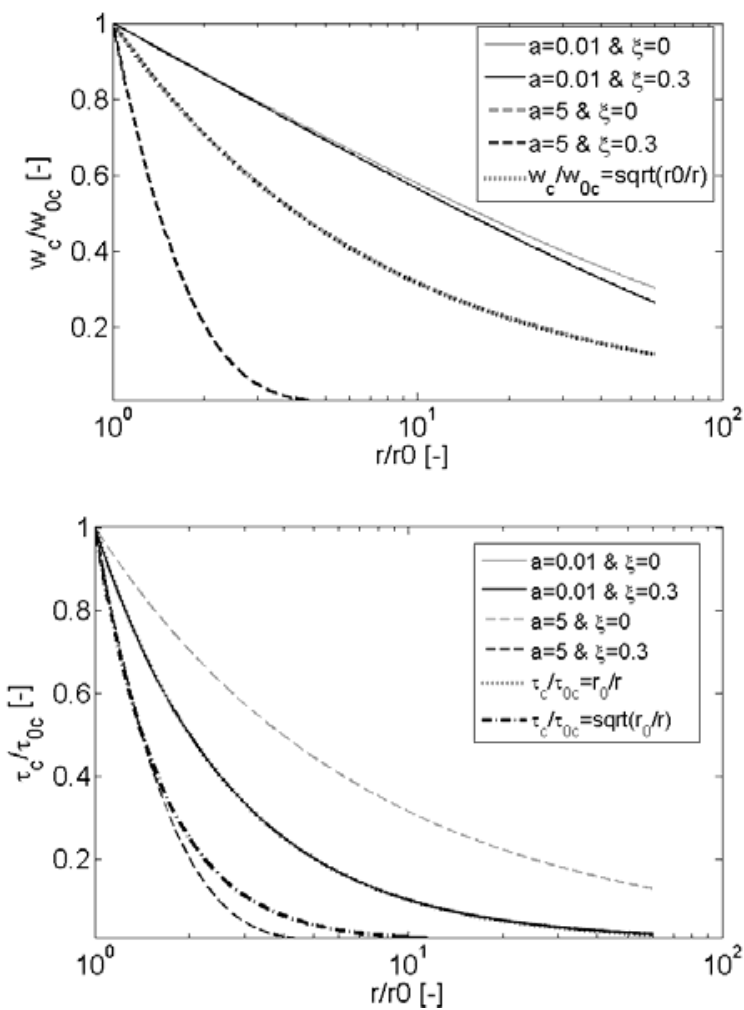

Fig. 11. Radial distribution of displacement and shear stress amplitudes

Impedance parameters for use in the Winkler approach have been studied by many researchers in the past. First models (e.g. Novak [14]) were based on the assumptions that the soil behavior is governed by the laws of (viscous-) elasticity and the soil is perfectly bonded to the pile. In practice however the soil region immediately adjacent to the pile can undergo a large degree of straining which causes the soil-structure system to behave non-linearly and even degrade under cyclic loading. Slippage can also occur about the contact area.

\section{Non linear aspects}

\subsection{Literature review}

Nonlinear models of axial pile-soil vibration started with the works of Novak and Sheta [15], Mitwally and Novak [16], Han and Sabin [17], and El Naggar and Novak ([18],[19]) who suggested distinguishing two separate radial soil zones around the pile shaft: an inner zone with reduced shear stiffness and an outer zone where the elastic solution is considered.

To eliminate undulations in the impedance functions due to wave reflections from the interface between the two media, some researchers proposed a continuously increasing modulus with radial distance to the pile shaft. Gazetas and Dobry [20] and Veletos and Dotson [21] suggested schemes in which the modulus increased unboundedly. Han and Sabin [17] formulated impedances based on a parabolic variation of the medium properties so that the inner zone has properties smoothly approaching those of the outer zone.

These contributions however address the problem of lateral soil heterogeneity with only qualitative reference to the non-linear soil response, since the variations of soil properties invoked are merely hypothetical. To aid practical applications, Michaelides et al. ([22],[23]) utilized experimental data (e.g. Vucetic \& Dobry [8]) characterizing the dependence of the secant shear modulus and hysteretic damping of soil on the shear strain amplitude and the nature of the soil (the latter represented by the plasticity index PI). The variation of modulus and damping is then related to the magnitude of the applied load through the amplitude of the shear strains induced within a succession of co-axial cylinders. Such an approach involves assumptions related to the shear stress distribution and implies the use of an iterative procedure to calculate the variation of modulus as a function of the distance to the pile shaft.

Some modifications to the Michaelides et al.'s model have been proposed by Holeyman et al. [24] to simplify definitions of the model parameters as well as calculation procedures. Using the modified method, a refined soil discretization can be achieved based on more rigorous soil behavior description and without a priori assumptions about the shear modulus or shear stress radial distributions.

The following sections describe analytical solutions assuming various theoretical radial variations of shear modulus. The results are evaluated over a range of parameters and compared with those obtained from the semi-analytical model derived from [22] and [23], and from a radial discrete model simulating the pile shaft and soil movements by integrating of the laws of motion ([25], [26], [27] (pile driving model), [9], [28], and [29] (Vibratory pile driving)).

\subsection{Radius-dependent shear modulus}

Assuming that the stress developed into the soil attenuates according to the inverse of the radial distance, 
which is exact in the static case, the following can be established: $\tau / \tau_{0}=r_{0} / r$. If we further consider that the stress $\tau_{0}$ is a fraction $f$ of the shear strength $\tau_{\max }$, i.e. $\tau / \tau_{\max }=f . r_{0} / r,(5)$ becomes:

$G_{s}=G_{\max } \cdot\left(1-f .\left(\frac{r_{0}}{r}\right)\right)$

where $f$ can be viewed as a "loading factor" or soil strength mobilization ratio at $r=r_{0}$ : it is actually the inverse of the factor of safety of the pile shaft capacity.

Because of the variation of the shear modulus with the radial distance, two extreme shear wave velocities can be distinguished: $V_{s 0}=\sqrt{G_{s 0} / \rho}$ at the pile shaft-soil interface and $V_{f f}=\sqrt{G_{\max } / \rho}$ in the free field at the furthest distance away from the pile. Dimensionless frequencies can thus be defined respectively for the pile shaft-soil interface: $\quad a_{0}=\omega \cdot r_{0} / V_{s 0} \quad$ (or $\left.a_{0}^{*}=a_{0} / \sqrt{1+2 i \xi}=\omega . r_{0} / V_{s 0}^{*}\right)$ and the free-field: $a_{f f}=\omega . r_{0} / V_{f f} \quad\left(\right.$ or $\left.a_{f f}^{*}=a_{f f} / \sqrt{1+2 i \xi}\right)$. Defining $\zeta=r / r_{0}>1$ the dimensionless distance to the pile shaft, the general equation of movement becomes:

$\frac{\partial^{2} w_{c}}{\partial \zeta^{2}}+\frac{1}{\zeta} \cdot \frac{\zeta}{\zeta-f} \cdot \frac{\partial w_{c}}{\partial \zeta}+\left(a_{f f}^{*}\right)^{2} \cdot \frac{\zeta}{\zeta-f} \cdot w_{c}=0$

Analytical solutions of (21) are presented in [24] and discussed below. Influence of the ' $f$ ' parameter on the shear modulus distribution is depicted in figure 12. The reader is referred to Bertin [30] for solutions based on other assumptions of shear modulus distributions.

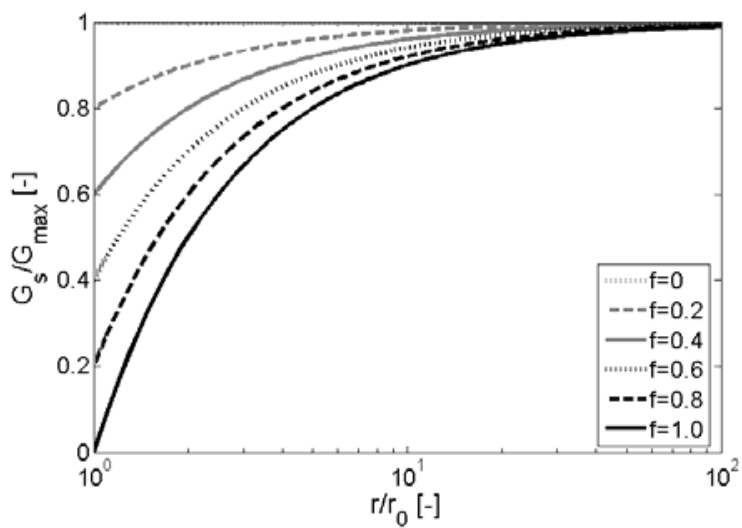

Fig.12. Influence of ' $f$ ' parameter on the shear modulus distribution, assuming stress attenuation according to $r^{-1}$

\subsection{Semi-analytical solutions}

Michaelides et al. [22],[23] suggested the use of a radial discretization and approximation of the shear modulus $\mathrm{G}_{\mathrm{s}}$ distribution within each zone using the following expression
$G=G_{s 0}^{*} \cdot\left(\frac{r}{r_{0}}\right)^{m}$

with $\mathrm{m}$ values decreasing with the distance to the pile shaft. This discretization also allows taking into account damping coefficients $\xi$ varying with $r$, albeit through a piecewise approximation.

Michaelides et al. assume that the shear stress distribution is independent from the shear modulus distribution, in order to alleviate interdependence between $G_{s}, \tau_{c}$ and $\gamma_{c}$. Based on this assumption and the use of empirical rules for the shear modulus distribution, they proposed following equation:

$G_{s}=G_{\max } \cdot\left(1-\left\{\Lambda \cdot \frac{r_{0}}{r} \cdot h\left(a_{r}\right)\right\}^{0.72}\right)$

where $\Lambda=\tau / \tau_{\max }$ is a loading intensity factor and $h\left(a_{r}\right)$ a shape function. Since the method imposes the use of iterations to determine $h\left(a_{r}\right)$ values, Michaelides limited the number of radial increments to four. That approximation has been further enhanced by Bertin [30], who developped a special routine able to iterate on many more radial increments.

Because of the limitations of the Michaelides et al.'s method, Bertin [30] suggested another approach based on analytical elements to discretize the radial coordinate. The shear modulus distribution is still described by a set of parabolas, but based on the Hardin \& Drnevich [4] functions and Masing rules [5]. Furthermore, a higher number of radial steps, up to 270 , is considered. Because values of $\mathrm{m}$ and $\xi$ are different for each element, displacements and stresses are obtained by assuring continuity of displacement and stress equilibrium. For each discretization step, the following equations (24) are used:

$$
w_{c}(r)=\left\{\begin{array}{c}
\zeta_{(i-1)}^{m_{0} / 2} \cdot\left[\begin{array}{l}
A_{(i-1)} \cdot J_{\chi_{(i-1)}-1} \cdot\left(\chi_{(i-1)} \cdot \lambda_{0_{(i-1)}} \cdot \zeta_{(i-1)}^{1 / \chi_{(i-1)}}\right) \\
+B_{(i-1)} \cdot Y_{\chi_{(i-1)}-1} \cdot\left(\chi_{(i-1)} \cdot \lambda_{0_{(i-1)}} \cdot \zeta_{(i-1)}^{\left.1 / \chi_{(i-1)}\right)}\right.
\end{array}\right] \text { if } r \leq r_{i} \\
\zeta_{(i)}^{m_{1} / 2} \cdot\left[\begin{array}{l}
A_{(i)} \cdot J_{\chi_{(i)}-1} \cdot\left(\chi_{(i)} \cdot \lambda_{0_{(i)}} \cdot \zeta_{(i)}^{1 / \chi_{(i)}}\right) \\
+B_{(i)} \cdot Y_{\chi_{(i)}-1} \cdot\left(\chi_{(i)} \cdot \lambda_{(i)} \cdot \zeta_{(i)}^{1 / \chi_{(i)}}\right)
\end{array}\right] \text { if } r_{i} \leq r \leq r_{i+1}
\end{array}\right.
$$

The four integration constants $A_{(i-1)}, A_{(i)}, B_{(i-1)}$ and $B_{(i)}$ are deduced from continuity and equilibrium conditions, adopting previously described boundary conditions (imposed displacement and outer radiation).

As illustrated by figure 13, Bertin analytical elements approximation [30] emulating Michaelides et al.'s concept is correct for low values of the dimensionless frequency, but is more questionable for higher values. 

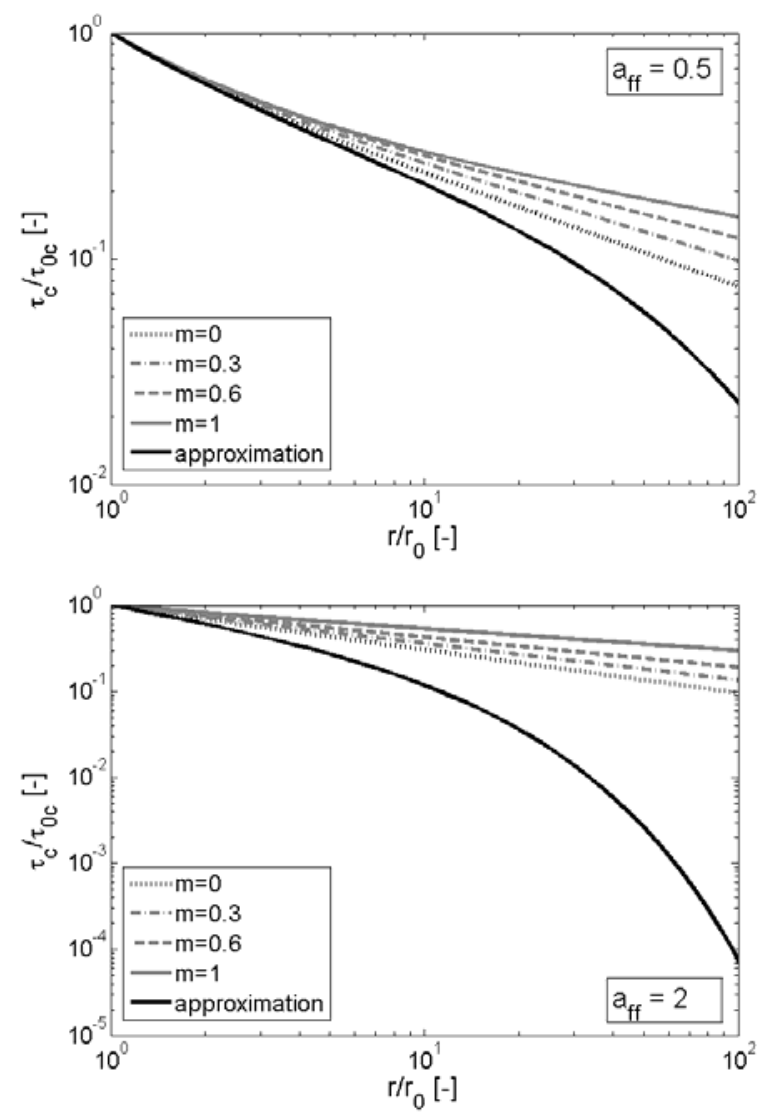

Fig. 13. Shear stress radial distributions for different values of (22) $m$ exponent compared to [30] enhanced approximation

\subsection{Numerical solution}

\subsubsection{Model}

Holeyman [26],[27],[28],[29] has suggested the use of a radial discrete model (see figure 14) to calculate the vertical shear waves propagating away from the pile shaft. The pile is considered as a rigid body and the soil is represented by discretizing the medium into concentric rings that have their own individual masses and that transmit forces to their neighboring ones. The movement of the pile and the rings is calculated from time integration of the law of motion: the equations of movement are integrated for each cylinder based on their dynamic shear equilibrium in the vertical direction. An energy absorbing boundary condition in accordance with plane-strain elasticity theory [14] limits the lateral extent of the model.

The model makes use of constitutive relationships representing the large-strain, dynamic and cyclic shear stress-strain strength behavior of the medium surrounding the pile shaft. Initially implemented for vibratory driving modeling in a Basic computer code "Hipervib-II" [9], it applied the hyperbolic Kondner law (4) and Masing rules [5] to model the shear force-displacement relationships between successive rings.

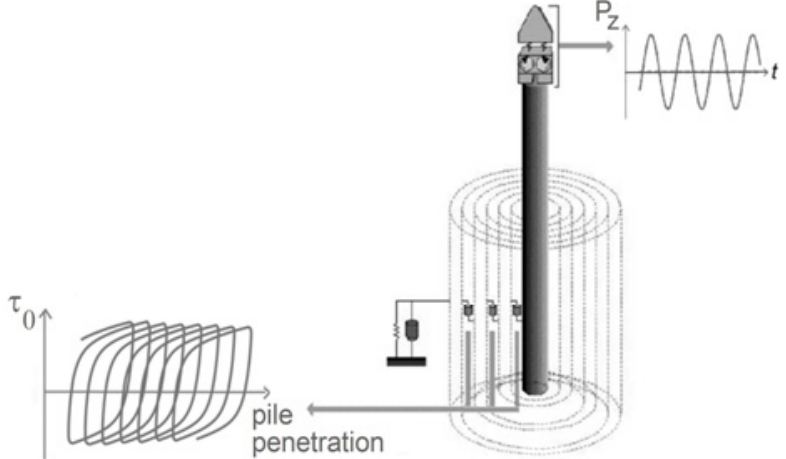

Fig. 14. Numerical model geometry (adapted from [28])

The program was further developed using Matlab ${ }^{\circledR}$ routines [30] to produce the results presented herein with a view to compare them with the above described semianalytical methods. Strain rate effects as well as cyclic degradation effects which are accounted for in the Hipervib-II program were however disabled to produce results compatible with the assumptions adopted in the other methods discussed in this paper.

When comparing Hipervib-II with the (semi-) analytical methods, following considerations are made: (a) only results corresponding to the steady state (after a few second of simulations) are presented herein, (b) impedance parameters are calculated as follows: $I_{z}=2 \pi \cdot r_{0} \cdot \frac{\int_{0}^{2 \pi / \omega} \tau\left(r_{0}, t\right) d t}{\int_{0}^{2 \pi / \omega} w\left(r_{0}, t\right) d t}$

with $w(t)$ imposed at the pile shaft boundary and $\tau(t)$ calculated by Hipervib-II at the pile shaft-soil interface. In order to compare the numerical results with the analytical ones presented herein for the shaft only, the base resistance that can be modeled in Hipervib-II has been set to zero.

\subsubsection{Radial distribution of shear modulus}

Relevant results are related to the evolution of the shear modulus as a function of the radial distance, by reference to its value at the pile shaft-soil interface. Figure 15 shows results obtained with three methods: the analytical solution to (21), Michaelides et al. original approximation (4 increments, labelled as "M-4"), and Hipervib-II program.

Table 1. Reference parameters for comparative study

\begin{tabular}{|l|l|l|}
\hline Soil density $(\rho)$ & 1.8 & $\mathrm{~T} / \mathrm{m}^{3}$ \\
\hline Maximum shear modulus $\left(\mathrm{G}_{\max }\right)$ & 60 & $\mathrm{MPa}$ \\
\hline Free field shear wave celerity $\left(V_{f f}\right)$ & 183 & $\mathrm{~m} / \mathrm{s}$ \\
\hline Shear strength $\left(\tau_{\max }\right)$ & 0.15 & $\mathrm{MPa}$ \\
\hline Reference shear strain $\left(\gamma_{r}\right)$ & 1.25 & $10^{-3}$ \\
\hline Hysteretic damping value $(\xi)$ & 0 & - \\
\hline Pile shaft radius $\left(\mathrm{r}_{0}\right)$ & 0.5 & $\mathrm{~m}$ \\
\hline
\end{tabular}



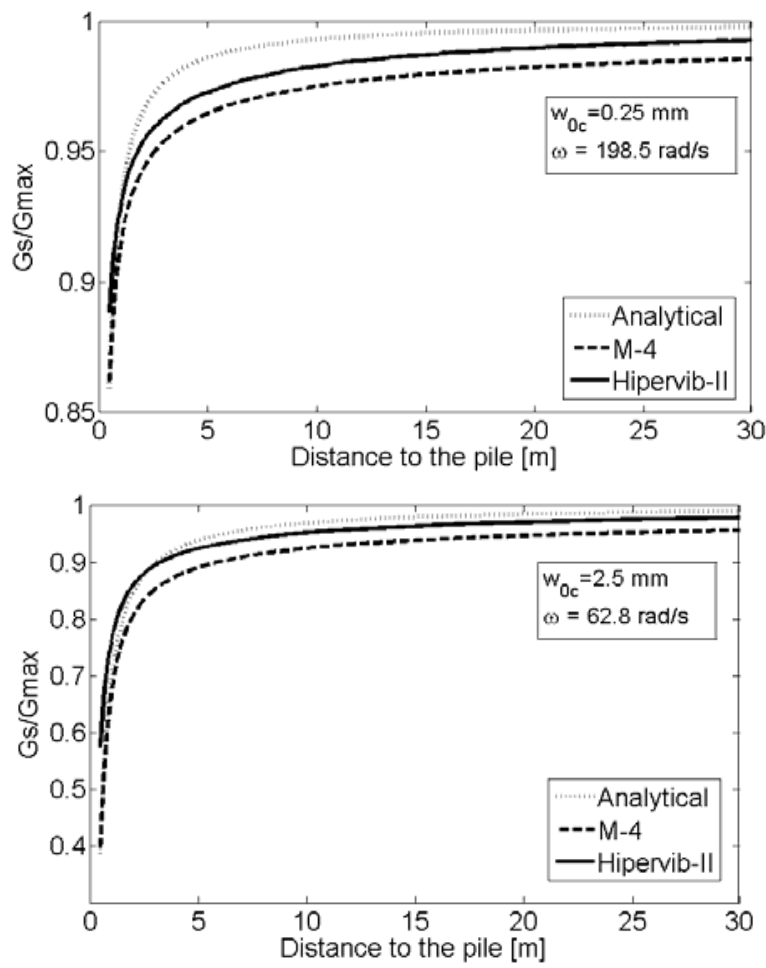

Fig. 15. Shear modulus distributions according to different approaches for same acceleration amplitude of $9.9 \mathrm{~m} / \mathrm{s}^{2}$

Reference parameters used to that end are summarized in Table 1, for two different combinations of imposed displacement amplitude and frequency leading to a given acceleration amplitude of $9.9 \mathrm{~m} / \mathrm{s}^{2}$.

For the same imposed acceleration amplitude $\left(w_{0 c} . \omega^{2}\right)$, all three methods indicate a more heavily degraded shear modulus at the pile shaft-soil interface for a larger displacement amplitude. When however compared to the Michaelides original method, the analytical results [30] better match those of the numerical model.

\subsection{Comparison of calculated impedance parameters}

Impedance parameters deduced from application of the semi-analytical method using Table 1 parameters are presented in figure 16 with a view to emphasize the influence of the imposed displacement amplitude. Figure 16 represents the real and imaginary parts of the impedance versus the dimensionless frequency $a_{f f}$ for three displacement amplitudes.

Comments made for the homogeneous model in Section 3.3 are also applicable to the non-homogeneous models, as far as the evolution of the impedance curves as functions of the dimensionless frequency is concerned, when trading the hysteretic damping coefficient for the amplitude of displacement. A comparison between semianalytical and analytical results is presented in figure 17, indicating some quantitative variations in the impedance results that mainly depend on the free field dimensionless frequency. In the above example, analytical results are quite close to the Bertin [30] approximation. If that
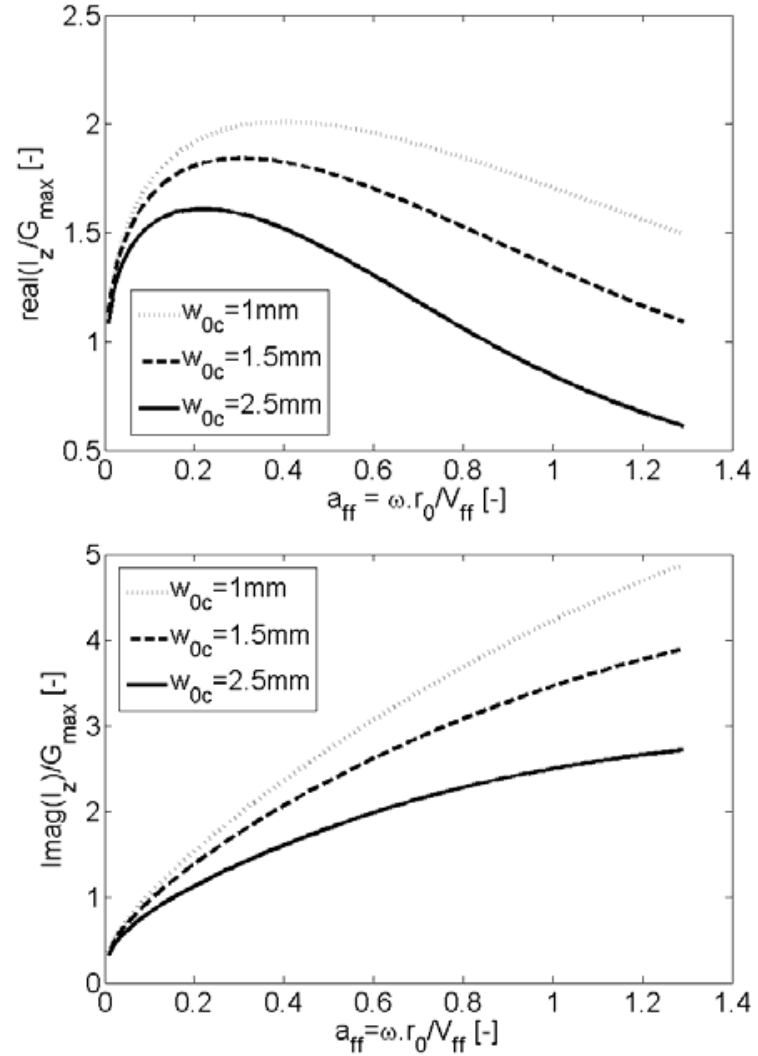

Fig. 16. Stiffness and damping parameters deduced from the semi-analytical method (see Table 1 for reference parameters)

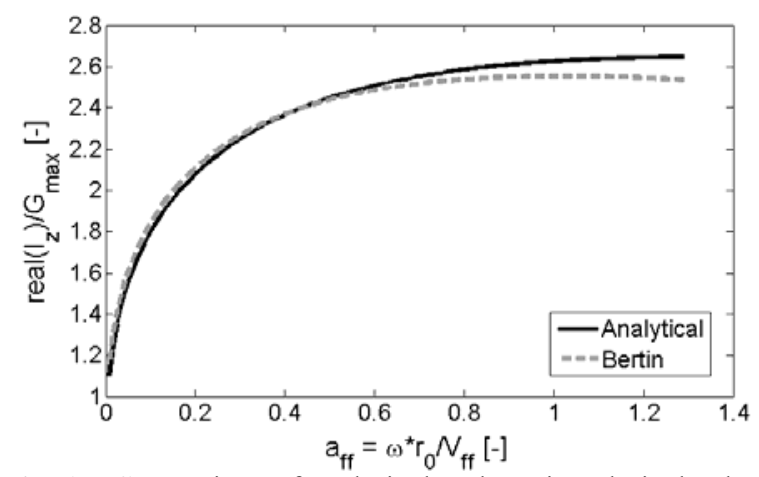

Fig. 17. Comparison of analytical and semi-analytical solutions for $\mathrm{w}_{\mathrm{oc}} / \mathrm{r}_{\mathrm{o}}=0.410^{-3}$ (see Table 1 for reference parameters)

observation could be extended to other values of $w_{o c} / r_{o}$ and $\xi$, it would mean that the simplest form of stress attenuation (corresponding to the static case) could be adopted for the dynamic cases.

Combined influences of frequency and imposed displacement amplitudes are represented in figure 18 for the analytical method. Both real and imaginary parts of the impedance decrease as the imposed displacement increases, whatever the frequency. Inspection of (12) and figure 19 shows how the stress value at the pile shaft-soil interface naturally tends towards the shear strength with increasing displacements, evidencing another manifestation of stiffness degradation.

The evolutions of the pile shaft friction mobilization ratio $\left(\tau_{o c} / \tau_{\max }\right)$ versus dimensionless displacement $\left(w_{o c} / r_{o}\right)$ shown in figure 19 can be compared to so-called 
"t-z curves" published in the literature to evaluate the pile shaft load-displacement behavior under axial static $\left(a_{f f}=0\right)$ conditions. As an example, static friction mobilization curves adopted by Holeyman [25] based on an extension of the influence radius $R_{m}$ approach suggested by Randolph and Wroth [31] to incorporate a hyperbolic stress-strain law are also plotted in figure 19 for two values of the boundary radius $R_{m}$. Confirmation of the effective reduction of the apparent "quake" value as velocity increases, observed by Holeyman [7] can be found in figure 19 for increasing values of $a_{f f}$.
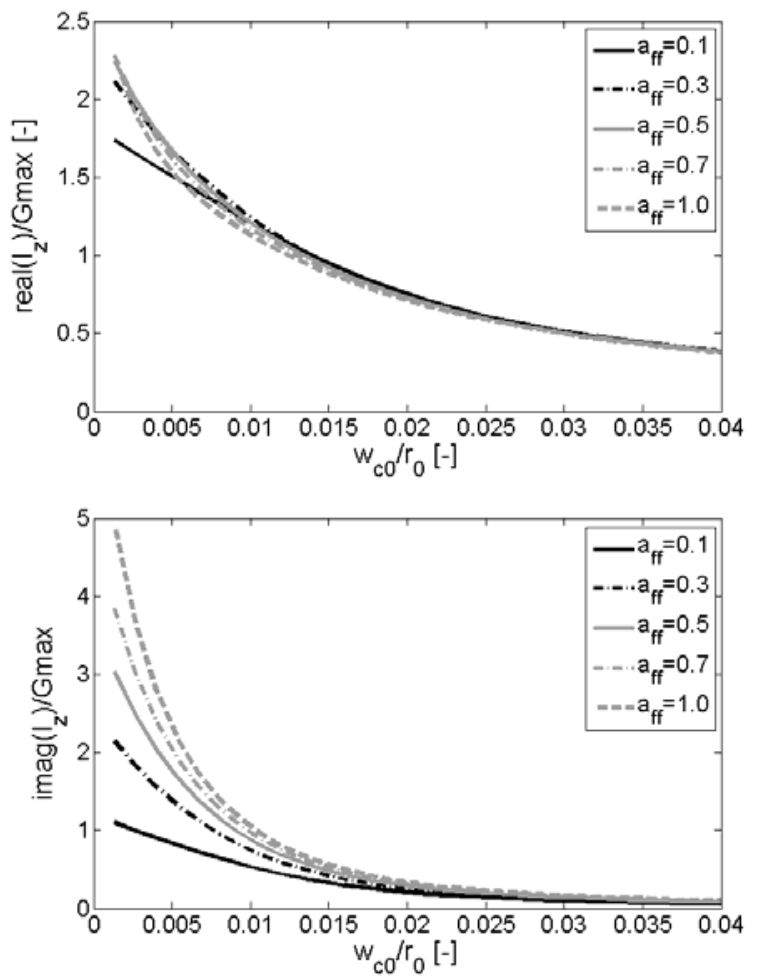

Fig. 18. Impedance parameters as functions of the imposed displacement (analytical model for Table 1 parameters).

\section{Conclusions}

The dynamic axial response of pile shafts can be approached using the concept of a continuously distributed mechanical impedance replacing the embedding medium. Equivalent impedance parameters can be defined to characterize the equivalent in-phase 'spring' and the equivalent out-of-phase 'dashpot'. When considering a pile shaft undergoing axial oscillations, shear strains (and shear stresses) are induced in the surrounding soil, the amplitude of which attenuates radially away from the pile shaft. Analytical equivalent linear methods and a numerical method are shown to adequately derive those amplitudes of shear stresses and shear strains as functions of the radial distance $r$ to the pile shaft. The corresponding dynamic impedance components are then readily determined.

The analytical solution proposed herein accommodates a continuous variation of soil properties alleviating wave reflections and avoiding numerical convergence problems. By contrast, the semi-analytical method suffers from numerical limitations arising from the radial soil discretization. The semi-analytical method correctly models a radial variation of the soil hysteretic damping, contrary to the analytical method which assumes a homogeneous hysteretic damping.

Similar approaches can be used to address the dynamic non linear response of piles under a lateral mode of deformation, as well as coupling effects between the axial and lateral modes of deformation [32].
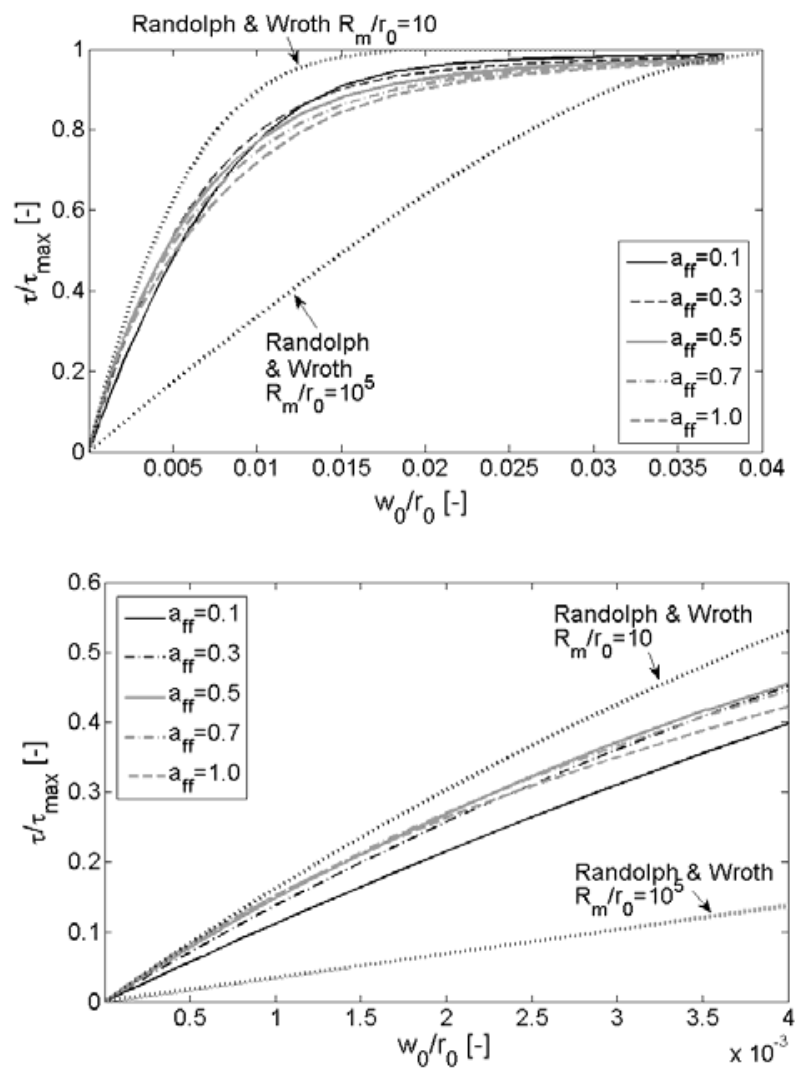

Fig. 19. Shear stress developed at the pile shaft-soil interface as a function of the imposed cyclic displacement amplitude (analytical model with Table 1 parameters vs. typical static t-z curve)

\section{Notations}

The following symbols are used in this paper:

$a=$ dimensionless frequency $\omega . r_{0} / V_{s}[-]$

$a^{*}=$ complex value of dimensionless frequency

$a / \sqrt{1+2 i \xi}=\omega \cdot r_{0} / V_{s}^{*}[-]$

$a_{0}=$ dimensionless frequency for the pile shaft-soil interface $\omega . r_{0} / V_{s 0}[-]$

$a_{f f}=$ dimensionless frequency in the free field $\omega . r_{0} / V_{f f}[-]$ 
$C_{z}=$ damping coefficient $\left[\mathrm{kPa} \cdot \mathrm{s}^{-1}\right]$

$C_{z a}=\mathfrak{I}\left\{I_{z}\right\} /\left(\omega . G_{s 0}\right)=$ dimensionless damping parameter [-]

$f=$ "loading factor" or soil strength mobilization ratio at $r=r_{0}[-]$

$G=$ shear modulus $[\mathrm{kPa}]$

$G^{*}=$ complex shear modulus $G .(1+2 i \xi)[\mathrm{kPa}]$

$G_{\max }=$ initial (maximal) shear modulus $[\mathrm{kPa}]$

$G_{s}=$ secant shear modulus $[\mathrm{kPa}]$

$G_{s 0}=$ shear modulus at the pile shaft-soil interface

$\left(r=r_{0}\right)[\mathrm{kPa}]$

$H_{n}^{2}=$ Hankel function $J_{v}-i Y_{v}$

$I_{0}=$ modified Bessel function of order 0 of first type

$I_{z}=$ unit (lineal) shear impedance of the soil against the pile shaft movement in the $\mathrm{z}$ direction $[\mathrm{kPa}]$

$J_{v}=$ Bessel function of order $v$ of first type

$k=$ shear wave number $\omega / V_{s}\left[\mathrm{~m}^{-1}\right]$

$k^{*}=$ complex shear modulus $\omega / V_{s}^{*}\left[\mathrm{~m}^{-1}\right]$

$k_{f f}^{*}=$ complex shear modulus in the free field $a_{f f}^{*} / r_{0}$ $\left[\mathrm{m}^{-1}\right]$

$K_{0}=$ modified Bessel function of order 0 of second type

$K_{z}=$ the stiffness coefficient $[\mathrm{kPa}]$

$K_{z a}=$ dimensionless stiffness $\mathfrak{R}\left\{I_{z}\right\} / G_{s 0}[-]$

$r=$ distance to the pile shaft $[\mathrm{m}]$

$r_{0}=$ pile shaft radius $[\mathrm{m}]$

$R_{m}=$ influence radius of shear strain field

$t=$ time $[\mathrm{s}]$

$V_{s}=$ shear wave velocity $\sqrt{G_{s} / \rho}\left[\mathrm{m} . \mathrm{s}^{-1}\right]$

$V_{s}^{*}=$ complex value of shear wave velocity $\sqrt{G_{s}^{*} / \rho}$ $\left[\mathrm{m} \cdot \mathrm{s}^{-1}\right]$

$V_{s 0}=$ shear wave velocity at the pile shaft-soil interface $\sqrt{G_{s 0} / \rho}\left[\mathrm{m} . \mathrm{s}^{-1}\right]$

$V_{f f}=$ shear wave velocity in the free field $\sqrt{G_{\max } / \rho}$ $\left[\mathrm{m} \cdot \mathrm{s}^{-1}\right]$

$w=$ displacement $[\mathrm{m}]$

$w_{c}=$ time-independent value of displacement [m]

$w_{o}=$ displacement at the pile shaft $[\mathrm{m}]$ $w_{o c}=$ amplitude of imposed displacement at the pile shaft $[\mathrm{m}]$

$Y_{v}=$ Bessel function of order $v$ of second type

$\gamma=$ shear strain $[-]$

$\dot{\gamma}=$ shear strain rate $\left[\mathrm{s}^{-1}\right]$

$\gamma_{c}=$ amplitude of shear strain [-]

$\gamma_{r}=$ reference strain $\tau_{\max } / G_{\max }[-]$

$\xi=$ damping coefficient [-]

$\rho=$ soil density $\left[\mathrm{T} / \mathrm{m}^{3}\right]$

$\tau=$ shear stress $[\mathrm{kPa}]$

$\tau_{c}=$ amplitude of shear stress $[\mathrm{kPa}]$

$\tau_{0}=$ shear stress at the pile shaft-soil interface $[\mathrm{kPa}]$

$\tau_{0 c}=$ amplitude of shear stress at the pile shaft-soil interface $[\mathrm{kPa}]$

$\tau_{\max }=$ maximal shear stress (shear strength) $[\mathrm{kPa}]$

$\omega=$ circular frequency $\left[\mathrm{rad} . \mathrm{s}^{-1}\right]$

$\varsigma=$ dimensionless distance to the pile shaft $r / r_{0}>1[-]$

The asterisk indicates that the viscous soil behavior (characterized by the Kelvin-Voigt formulation) is taken into consideration.

\section{References}

1. Upwind - Integrated Wind Turbine Design. Project funded by the European Commission under the $6^{\text {th }}$ (EC) RTD Project No. 019945 (SE6), (2010)

2. Smith E. A. L., Pile driving analysis by the wave equation. Journal of Soil Mechanics and Foundation. 86(4): 35-61, (1960)

3. Hardin, B0, Black, WL. Vibration modulus of normally consolidated clay. Journal of the Soil Mechanics and Foundations Division ASCE 1968; 92(2): 353-369

4. Hardin, B. O. and Drnevich, V. P., Shear modulus and damping in soils: design equations and curves Journal of the Soil Mechanics and Foundations Division, American Society of Civil Engineers 98 7, pp. 667-692, (1972)

5. Masing, G., Eigenspannungen und Verfeistigung beim Messing Proc. $2^{\text {nd }}$ Int. Congress of Appl.Mech., pp. 332-335 (1926)

6. Kondner, R. L., Hyperbolic Stress-Strain Response: Cohesive Soils. Journal of the Soil Mechanics and Foundations Division, ASCE, 89, No. SM1, pp.115143 (1963)

7. Ishibashi, I., \& Zhang, X., Unified dynamic shear moduli and damping ratios of sand and clay. Soils and Foundations, 33(1) , 182-191 (1993) 
8. Vucetic, M. \& Dobry, R., Effect of soil plasticity on cyclic response J. Geotech. Engng, ASCE 117, No. 1, 89-107 (1991)

9. Holeyman, A., HIPERVIB-II, A detailed numerical model proposed for Future Computer Implementation to evaluate the penetration speed of vibratory driven sheet Piles Research report for BBRI, EarthSpectives, Irvine, Ca, USA, 54p (1993)

10. Bauer, E., Calibration of a Comprehensive Hypoplastic Model for Granular Materials. Soils and Found. 36(1), 13-26 (1996).

11. Von Wolffersdorff, P. A., A hypoplastic relation for granular materials with a predefined limit state surface. Mechanics of Cohesive-Frictional Materials (1),251-271 (1996)

12. Sommerfeld, A., Mechanics, Lectures on Theoretical Physics, volume I. Academic press Inc., 4 edition (1952)

13. Storz, M., Chaotic Motion in Pile-Driving. First International Conference Soil Dynamics and Earthquake Engineering (pp. 503-512). Southhampton, England: Computational Mechanics (1991)

14. Novak M., Dynamic stiffness and damping of piles Can. Geotech. J. 11, No. 4, 574-598 (1974)

15. Novak, M. and Sheta, M., Approximate approach to contact effects of piles In Special technical publication on dynamic response of pile foundations: analytical aspects (eds M. W. O'Neill \& R. Dobry). New York: ASCE (1980)

16. Mitwally $H$ and Novak $M$, Pile driving analysis using shaft and FEM. Proceedings of the third international conference on the application of stress wave theory to piles; Bitech Publishers, Vancouver (1988)

17. Han Y.C. and Sabin G.C., Impedances for radially inhomogeneousviscoelastic soil media J. of Engrg. Mechanics, Vol.121, No.9, pp.939-947 (1995)

18. El Naggar, M. \& Novak, M., Nonlinear axial interaction in pile dynamics. Journal of Geotechnical Engineering, 120(4): 678-696 (1994)

19. El Naggar, M. \& Novak, M., Nonlinear model for dynamic axial pile response. Journal of Geotechnical Engineering, 120(2): 308-329 (1994)

20. Gazetas, G. and Dobry, R., Simple radiation damping model for piles and footings. J. Engrg. Mech., ASCE, 10(6), 937-956 (1984)

21. Veletsos, A. S. \& Dotson, K. W., Vertical and torsional vibration of foundations in inhomogeneous media J. Geotechnical Engineering Division, ASCE 114, No. 9, 1002-1021(1988)

22. Michaelides O., Gazetas G., Bouckovalas G. and Chrysikou E, Approximate nonlinear dynamic axial response of piles Geotechnique 48, No.1, 33-53 (1997)

23. Michaelides O., Bouckovalas G. and Gazetas G., Non-linear soil properties and impedances for axially vibrating pile elements Soils and foundations, Vol.38, No.3, 129-142 (1988)

24. Holeyman, A., Bertin, R., and Whenham, V., Impdedance of pile shafts under axial vibratory loads, Soil Dynamics and Earthquake Engineering, http://dx.doi.org/10.1016/j.soildyn.2012.09.006, 115-126 (2013)

25. Holeyman, A., Contribution à l'étude du comportement transitoire non-linéaire des pieux pendant leur battage. Doctoral thesis, Université Libre de Bruxelles, April, 1984, 584 p. (1984)

26. Holeyman, A., Dynamic non-linear skin friction of piles, Proceedings of the International Symposium on Penetrability and Drivability of Piles, San Francisco, 10 August, Vol. 1, pp. 173-176 (1985)

27. Holeyman, A., Technology of Pile Dynamic Testing, in: Application of Stress-Wave Theory to Piles, edited by F. Barends, Balkema, Rotterdam, pp. 195215 (1992)

28. Holeyman, A., and Legrand, C., Soil Modeling for Pile Vibratory Driving, U.S. FHWA International Conference on Design and Construction of Deep Foundations, Orlando, Florida, December 1994, Vol. II, pp. 1165-1178 (1994)

29. Holeyman, A., Vibratory Pile Driving, in: Quality Assurance on Land and Offshore Piling, Edited by S. Nyyama and J. Beim, Balkema Publishers, Rotterdam, 2000, pp.479-494 (2000)

30. Bertin R., Modélisation de l'interaction axiale solpieu - Détermination des paramètres d'impédance d'un milieu non-homogène Master's thesis, Université Catholique de Louvain (2009)

31. Randolph, M.F. and Wroth, C.P., Analysis of deformation of vertically loaded piles, J. Geotech. Eng. Div. ASCE 104(GT12): 1465-1488 (1978)

32. Malek, A. and Holeyman, Flexural Analysis in Dynamic Pinned Head Pile Testing, Geotech Geol Eng 32:59-70 (2014)

33. Malek, A. and Holeyman, A., Numerical evaluation of nonlinear lateral pile vibrations on nonlinear axial response of pile shaft Soils and foundations, http://dx.doi.org/10.1016/j.sandf.2013.04.002, (2013) 